\title{
Performance Evaluation of 5G Millimeter-Wave Cellular Access Networks Using a Capacity-Based Network Deployment Tool
}

\author{
Michel Matalatala, Margot Deruyck, Emmeric Tanghe, Luc Martens, and Wout Joseph \\ Department of Information Technology, Ghent University/iMinds, Technologiepark-Zwijnaarde 15, 9052 Ghent, Belgium \\ Correspondence should be addressed to Michel Matalatala; michel.matalatala@intec.ugent.be
}

Received 21 September 2016; Accepted 30 November 2016; Published 10 January 2017

Academic Editor: Michal Choras

Copyright ( 2017 Michel Matalatala et al. This is an open access article distributed under the Creative Commons Attribution License, which permits unrestricted use, distribution, and reproduction in any medium, provided the original work is properly cited.

\begin{abstract}
The next fifth generation (5G) of wireless communication networks comes with a set of new features to satisfy the demand of dataintensive applications: millimeter-wave frequencies, massive antenna arrays, beamforming, dense cells, and so forth. In this paper, we investigate the use of beamforming techniques through various architectures and evaluate the performance of $5 \mathrm{G}$ wireless access networks, using a capacity-based network deployment tool. This tool is proposed and applied to a realistic area in Ghent, Belgium, to simulate realistic $5 \mathrm{G}$ networks that respond to the instantaneous bit rate required by the active users. The results show that, with beamforming, $5 \mathrm{G}$ networks require almost $15 \%$ more base stations and 4 times less power to provide more capacity to the users and the same coverage performances, in comparison with the $4 \mathrm{G}$ reference network. Moreover, they are 3 times more energy efficient than the $4 \mathrm{G}$ network and the hybrid beamforming architecture appears to be a suitable architecture for beamforming to be considered when designing a $5 \mathrm{G}$ cellular network.
\end{abstract}

\section{Introduction}

The increasing demand of applications in terms of throughput and latency explains the evolution of telecommunication standards. The next generation of telecommunication standards such as the 5th-generation (5G) wireless communication networks are expected to considerably accommodate larger number of wireless connections to better support existing and evolving applications including social media, high definition video streaming, full-featured web browsing, and real-time gaming. This can be made possible thanks to new features utilized in $5 \mathrm{G}$ wireless access networks as presented in [1, 2]: massive MIMO (Multiple Input Multiple Output), beamforming, small dense networks, millimeterwave frequency, and movable base stations. However, the fast growing data traffic volume and dramatic expansion of network infrastructure will inevitably trigger tremendous escalation of energy consumption in wireless networks. This will directly result in the increase of green house gas emission and pose ever increasing urgency on the environmental protection [3].

In this study, a capacity-based network deployment tool is proposed to meet the requirement of designing energy efficient 5G wireless networks, while providing at the same time the higher throughput required by the users. A similar method was used in [4] but was limited to the design of an energy efficient LTE-advanced network at $2.6 \mathrm{GHz}$, with its three main features: carrier aggregation, heterogeneous networks, and MIMO technology. Here, beamforming is implemented and investigated in order to assess its potential by means of system level simulations, thanks to the use of multiple antennas at the base station. Beamforming increases the base station antenna gain and help focusing antennas' energy in a desired direction, while preventing interference from others $[1,2]$. The assessment will consist in examining the influence of the use of beamforming technology on the overall network power consumption, network coverage, and network capacity. A realistic suburban case in Ghent, Belgium, is considered for this study.

The rest of this paper is organized as follows: Section 2 discusses the new features of the $5 \mathrm{G}$ wireless access networks, the power consumption models of its base station, and the energy efficiency metrics. In Section 3 , the network deployment tool is described and finally Section 4 presents the results obtained with the deployment tool with respect 
to the beamforming technology. We then provide the final conclusions in Section 5.

\section{5G Wireless Access Networks}

Research on next-generation 5G wireless systems, which aims to resolve several unprecedented technical requirements and challenges, has attracted growing interests from both academia and industry in the past few years. More than 5 billion devices demand wireless connections that run voice, data, and other applications in today's wireless networks [5].

The need for more capacity is just one key driver for mobile networks to evolve towards 5G. For the data-intensive applications to be working smoothly, the industries and academia agree on the following technical requirements for the $5 \mathrm{G}$ wireless network, which is not standardized yet [6]:

(i) Coverage and Data Rate. 5G should maintain connectivity anytime and anywhere with a minimum user experience data rate of $1 \mathrm{Gbps}$ [7].

(ii) Latency. The latency requirement is usually more difficult to achieve compared to that of the data rate as it demands that the data be delivered to the destination within a given period of time. For the 5G wireless network, the end-to-end latency requirement is expected to be in the order of $1-5 \mathrm{~ms}[8,9]$.

(iii) Connected Devices. The future $5 \mathrm{G}$ network will have the ability to incorporate huge number of connected devices, allowing them to reach up to 100 times the data rate of the $4 \mathrm{G}$ wireless network.

(iv) Multiple Radio Access Technologies. The 5G network is not meant to replace the current wireless networks. It will be built upon the existing wireless technologies: the Global System for Mobile Communications (GSM), third generation of mobile communications (3G), high-speed packet access (HSPA), long term evolution (LTE), long term evolution advanced (LTEA), and wireless fidelity (WiFi) $[8,9]$.

(v) Energy and Cost Efficiency. 5G wireless networks have to be designed to meet the requirements of dataconsuming applications but at a lower cost and higher energy efficiency, compared to the existing wireless networks [8].

Given the above requirements, the following new technologies will have to be enabled to make $5 \mathrm{G}$ wireless networks reality [1]:

(i) Millimeter-Wave Frequency. As the frequency spectrum under $6 \mathrm{GHz}$ is highly utilized, it appears that millimeter wave is the best candidate to respond to the huge requirements in terms of network capacity and from Gigabit broadband applications [10].

(ii) Massive MIMO. This is the extension of the MIMO technology used in $4 \mathrm{G}$ technology to a large number of antennas. Massive antenna arrays are put together to provide the ability to exploit the maximum possible degrees of freedom available in the spatial domain.
Therefore, it becomes possible to focus these energies towards a desired direction, while preventing propagation in the nondesired direction (beamforming). This can easily be achieved at millimeter-wave frequency since this high carrier frequency requires antenna elements to be very small, allowing the use of many antennas at both the base station (BS) and the mobile station (MS) [11]. There are mainly three types of beamforming architectures that are widely investigated: the digital beamforming (DBF), the analog or radio frequency beamforming $(\mathrm{ABF})$, and the hybrid beamforming architecture (HBF) [12]:

(1) Digital beamforming: this is the type of beamforming architecture whereby it is assumed that a transceiver is behind every antenna. So, the entire array processing is performed at the baseband side.

(2) Analog beamforming: this is the type of beamforming whereby the control of MIMO and beamforming is performed at radio frequency (RF) level. Here, a transceiver is assumed to drive the antenna array and the transmit and receive array processing is performed with $\mathrm{RF}$ components having phase shifting and potentially gain adjustment capabilities as well.

(3) Hybrid beamforming: the control of MIMO and beamforming is split between RF and baseband. Each set of antenna elements is driven by a transceiver. A hybrid architecture can use two to eight transceivers to drive the antenna array.

(iii) Small Cells. To satisfy the increasing traffic demands due to the growing number of users, densification of the infrastructure whereby $5 \mathrm{G}$ small cells are introduced in the $4 \mathrm{G}$ macrocell network is set to be a priority aspect in $5 \mathrm{G}$ communications [1].

This paper emphasizes the use of beamforming technology in the millimeter-wave frequency bands and investigates its influence on the behavior of the 5G wireless communications networks. The densification will serve as an another study in the future work.

\section{Capacity-Based Network Deployment Tool}

3.1. Design of the Network Deployment Tool. The network deployment tool is designed and proposed to meet the requirements of $5 \mathrm{G}$ wireless network. The choice for the design of such a tool is motivated by the fact that it simulates a realistic network that responds to the instantaneous bit rate (voice or data) required by the active users, in the considered area. This tool is used to simulate the different scenarios of $5 \mathrm{G}$ wireless communication networks, based on our assumptions, and the results are discussed and compared with the reference scenario. The following lines discuss the different steps of the simulation tool/algorithm.

3.1.1. Creation of the Traffic. The different steps leading to the creation of traffic files are indicated in Figure 1. First, a 


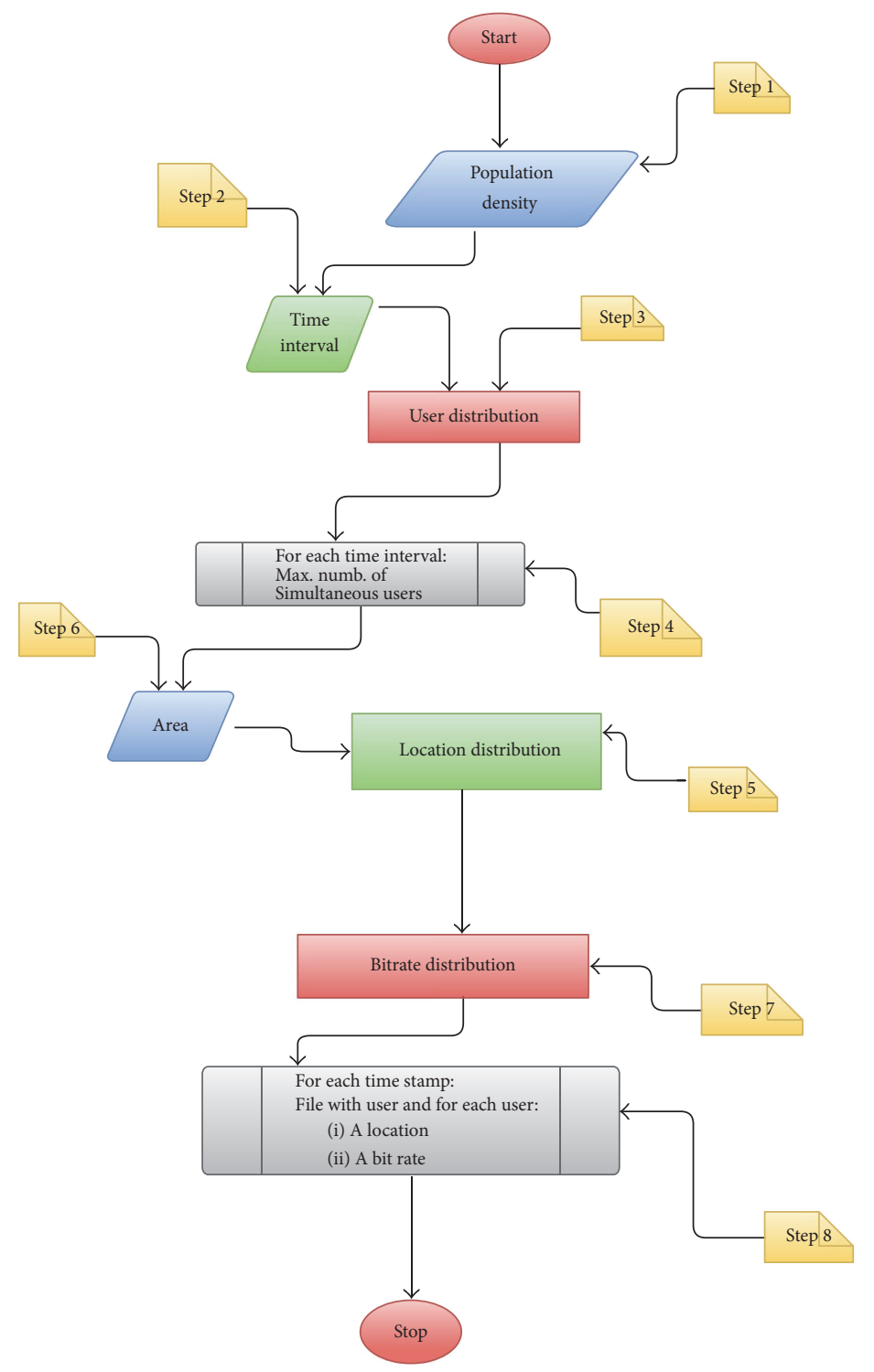

FIGURE 1: Flowchart of the creation of traffic.

traffic file is generated, containing, for each time interval, the maximum number of simultaneous active users. The location of the users in the considered area and the required bit rate are determined. All this information is gathered in a single file for each time interval; there are as many traffic files as the number of simulations. Various distribution functions are put together to produce these traffic files:

(i) User distribution (Figure 1, step 3): for a requested bit rate (voice and data), this distribution returns the maximum number of simultaneous active users. A distribution based on the confidential data retrieved from a Belgian mobile operator in Ghent has been used, which depends on the population density of the selected area [4].

(ii) Location distribution (Figure 1, step 6): the location of each user within the selected area of simulation is here defined. A uniform distribution is considered, meaning that each location in the area has the same chance to be chosen as a user location [4].

(iii) Bit rate distribution (Figure 1, step 7): based on the confidential data from the mobile operator, this distribution returns the bit rate that the individual user demands for its service. It is assumed that some users making voice calls at $64 \mathrm{kbps}$ and those requesting data transfer need $1 \mathrm{Mbps}$ [4].

Other higher bit rate distribution might be used for the future $5 \mathrm{G}$ services. It would require the use of massive MIMO technology to achieve these performances. Here, we have based our analysis on the constraints and data provided by a Belgian operator: the same area of interest, the same environment, the same base station, and user bit rate distributions. This will lead to a fair and realistic comparison between the two technologies. 


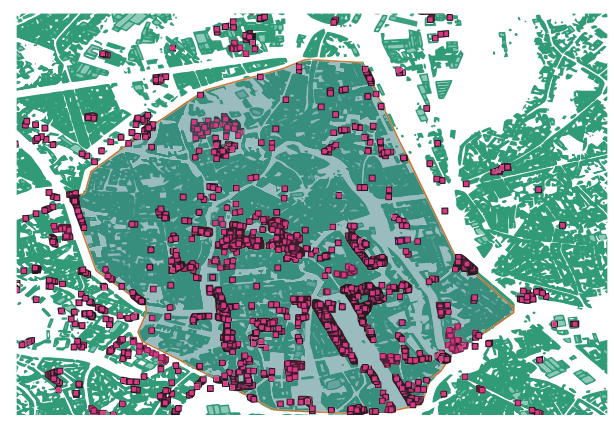

Figure 2: Selected area in Ghent, Belgium, and the possible location of the base stations.

3.1.2. Urban Environment. In this study, a suburban area of $6.85 \mathrm{~km}^{2}$ in Ghent, Belgium, has been used for the simulations (Figure 2). The following reference scenarios have been considered:

(i) Scenario I (reference): $4 \mathrm{G}$ network at $2.6 \mathrm{GHz}$, with $20 \mathrm{MHz}$ bandwidth without MIMO.

(ii) Scenario II: $5 \mathrm{G}$ network at $60 \mathrm{GHz}$. The bandwidth will be set at $500 \mathrm{MHz}$.

(1) Scenario II.a: 5G network without beamforming.

(2) Scenario II.b: $5 \mathrm{G}$ network with beamforming implemented at the base station only. The number of antennas will be varied from $8,16,32,64$, and then 256.

(3) Scenario II.c: $5 G$ network with beamforming implemented at both the base station and the mobile station. The number of BS antenna elements will be changing from $8,16,32,64$, and then 256, while on the MS side, the number of antenna elements will be set to 4 .

We would like to emphasize that these scenarios will focus on beamforming only, based on the above assumptions. The scenarios whereby the $5 \mathrm{G}$ services require high data rates are not examined, since it will require the combination of multiuser massive MIMO technology with beamforming in our analysis. Massive MIMO is a key enabling technology of $5 \mathrm{G}$ meant to increase the spectral efficiency by allowing parallel transmissions of user data to match with dataintensive applications [11]. This concept falls out of the scope of this study and will be examined in our further research on $5 \mathrm{G}$.

The scenarios are summarized in Table 1. To ensure at least $96 \%$ of $5 \mathrm{G}$ users are served, we extended the set of the possible locations of the base stations belonging to an existing Belgian mobile operator. They are indicated with red squares in Figure 2. For a better comparison with the $4 \mathrm{G}$ reference network, we assume the distributions described in Section 3.1.1. It is worth mentioning that the simulations have been carried out with the most simultaneous users present, which corresponds to the worst-case scenario. So, a time interval of 36 minutes has been used to allow the processing of 40 simulations within the 24-hour period (1440 minutes), resulting in the creation of 40 realistic networks. These 40 networks are needed for this time interval to obtain a good estimation of the different parameters. For analysis purposes, the mean value $(A v)$ and the 95th percentile (95p) of the investigated parameters will be considered. This applies to the number of required BSs, the total network power consumption, the capacity, and the coverage provided by the network.

3.1.3. Generation of the Network. The algorithm shown in Figure 3 is used to generate many $5 \mathrm{G}$ networks in such a way that energy efficiency is guaranteed. The $5 \mathrm{G}$ network will be created, for each time interval, based on the traffic file above (Figure 3, steps 1 and 2). Additional input files are needed here: a file containing the extended set of possible locations of the base stations in the considered area, a shape file describing the buildings (location, height, etc.) in the considered environment, a file defining all the link budget parameters for 5G, and, finally, a file consisting of all the typical power consumption values for the different components of the base station. The objective of the algorithm is to provide coverage for each user in the selected area, while optimizing the power consumption of the network. For each time interval of 1 hour, 40 simulations are processed whereby 40 networks are created. In total, for the $24 \mathrm{~h}$ time interval, 960 networks will be generated (Figure 3, step 2). The algorithm evaluates the distance between the new user (Figure 3, step 3) in the considered area and the already enabled base station. Based on this distance, the path loss experienced from that enabled base station is calculated and checked whether it is lower than the maximum allowable path loss (MAPL). The latter is the maximum path loss that a transmitted signal can be exposed to while still having sufficient strength at the receiver side to offer the bit rate requested by the user [4]. Of course, depending on the environment, a line-of-sight (LOS) or nonline-of-sight (NLOS) path loss model needs to be used as buildings may block the line of sight between the user and the base station (Figure 2). If the obtained path loss is lower than the MAPL, the new user will be connected on the existing active base station (Figure 3, step 4). Otherwise, if it is not possible to connect to an active one, a new base station will be switched on such that the path loss the user experiences is the lowest one among all the disabled base stations (Figure 3, step 5). At the same time, it should be lower than the MAPL for the user to benefit from the bit rate he requested for his services.

Furthermore, in Figure 3, step 6, the algorithm will check whether users already connected to other base stations can be migrated to this newly enabled base station, since they may experience a lower path loss from this base station. If this is the case, then there is a possibility to reduce the input power of the base station the user is removed from. If no base station can be enabled or all base stations are already active, the user cannot be served. This operation is repeated for all the users defined in the traffic file for a given time interval.

3.2. Calculation of the Path Loss at Millimeter Waves. The calculation of both the path loss and the MAPL is based on 
TABLE 1: Simulation scenarios.

\begin{tabular}{|c|c|c|c|c|c|}
\hline Scenarios & Beamforming support & Bandwidth [MHz] & Frequency $[\mathrm{GHz}]$ & $\begin{array}{c}\text { Number of antennas } \\
\text { at BS }[-]\end{array}$ & $\begin{array}{c}\text { Number of antennas } \\
\text { at MS [-] }\end{array}$ \\
\hline $4 \mathrm{G}$ ref. scenario I & No & 500 & 2.6 & 1 & 1 \\
\hline 5G scenario II.a & No & 500 & 60 & 1 & 1 \\
\hline 5G scenario II.b & At BS side only & 500 & 60 & $8,16,32,64$, and 256 & 1 \\
\hline 5G scenario II.c & At both BS and MS & 500 & 60 & $8,16,32,64$, and 256 & 4 \\
\hline
\end{tabular}

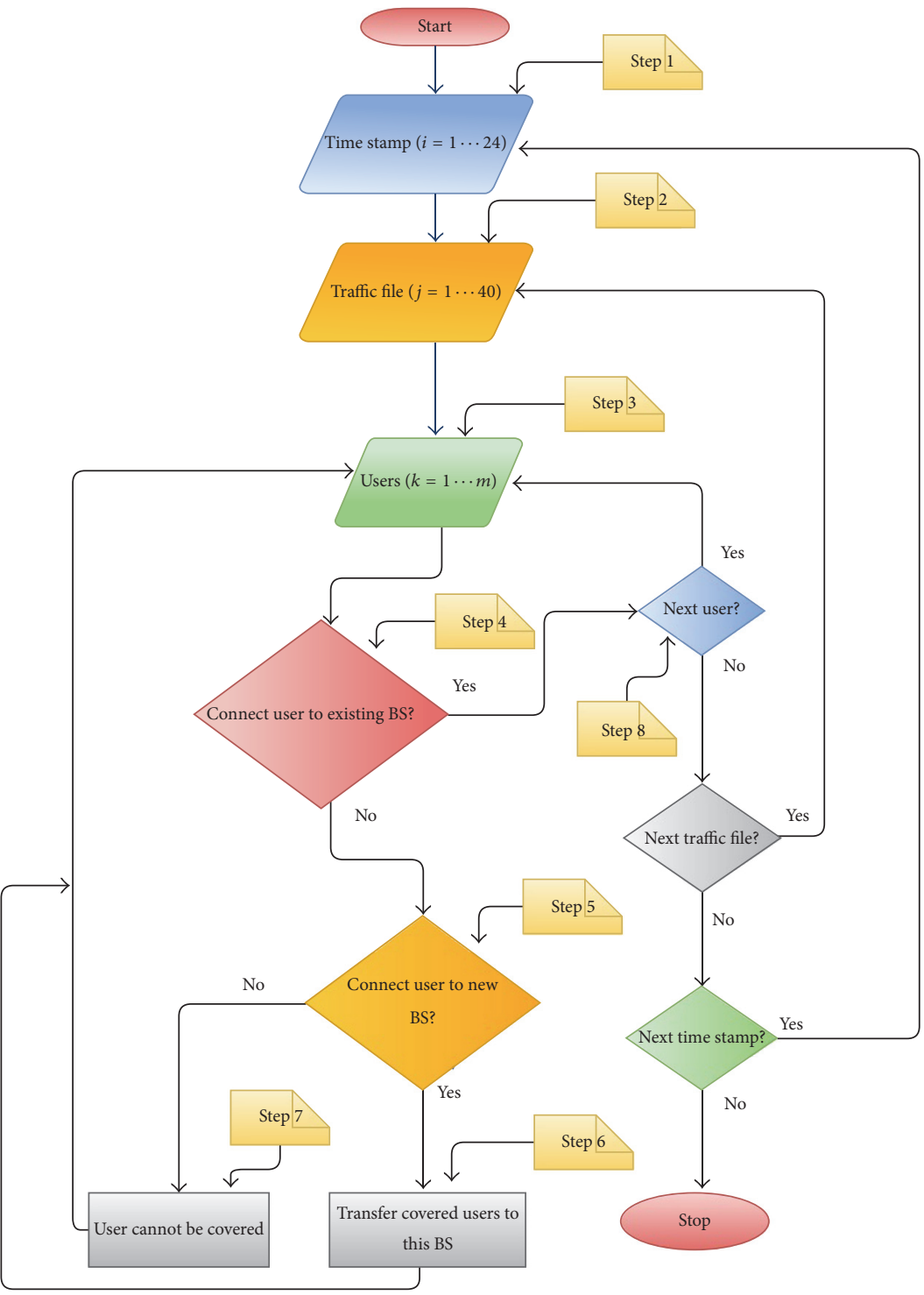

FIGURE 3: Network generation flowchart.

the main link budget parameters for $5 \mathrm{G}$ listed in Table 2 [1318]:

(i) The beamforming (BF) gain reflects the effect of the use of the multiple antennas at both the base station and the mobile station side. It is defined similarly as $10 \times \log _{10}(N)$ [19], where $N$ is the number of antenna elements at the side where BF is applied. In other words, if BF is applied at the BS side, then $N$ is the number of antennas used at the BS side, and if the MS is concerned, $N$ is the number of antennas at the MS side. Then, the total antenna array gain is calculated as the sum of the aforementioned beamforming gain and the singular antenna element gain [19].

(ii) Millimeter-wave atmospheric loss: this parameter accounts for the level of millimeter electromagnetic energy absorbed by gases like oxygen or attenuated by rain or foliage. In this study, we assume a constant value of $3.2 \mathrm{~dB}$ for the atmospheric loss at $60 \mathrm{GHz}$ 
TABLE 2: 5G link budget parameters.

\begin{tabular}{lc}
\hline Parameters & Values \\
\hline Carrier frequency & $60 \mathrm{GHz}$ \\
Channel bandwidth & $500 \mathrm{MHz}$ \\
Transmit antenna element gain & $10 \mathrm{dBi}$ \\
Transmit array antenna feed loss & $3 \mathrm{~dB}$ \\
TX power per base station antenna & $10 \mathrm{dBm}$ \\
Number of receive antenna array elements & 4 \\
Receive antenna element gain & $6 \mathrm{dbi}$ \\
SNR & $(7.39,15.4,17.5) \mathrm{dB}^{1}$ \\
Path loss exponent & 3.5 \\
mmWave penetration loss & $2 \mathrm{~dB}$ \\
mmWave atmospheric loss & $3.2 \mathrm{~dB}$ \\
Implementation loss & $3 \mathrm{~dB}$ \\
RX noise figure & $7 \mathrm{~dB}$ \\
Other losses (shadow, fading) & $20 \mathrm{~dB}$ \\
\hline
\end{tabular}

${ }^{1}$ Values of signal-to-noise ratio corresponding to [1/2 BPSK, 1/2 QPSK, and 1/2 16-QAM] [20].

[20]. This parameter introduces an additional attenuation at millimeter waves that must be accounted for since it increases the path loss and therefore reduces the range of the cell.

(iii) The path loss model: various path loss models related to the millimeter-wave frequency bands are proposed in the literature: the close-in reference distance path loss model [21], the floating-intercept path loss model [21], the alpha-beta-gamma path loss models [22], the Stanford University Intermediate (SUI) path loss models [23], and so forth. In this study, we assume the close-in reference distance path loss model since it is not an empirical model and it offers a substantial simplicity and a reasonable accuracy across many environments and frequency bands [24]. It is applicable to any type of terrain (urban, suburban, or rural) and the propagation coefficient, be it line-ofsight (LOS) or non-line-of-sight (NLOS), expressed through the path loss exponent. The latter takes into account the separation distance between the transmitter and the receiver and the heights of their respective antennas. Equation (1) determines the path loss $\mathrm{PL}(d)($ in $\mathrm{dB})$ at a distance $d$ :

$$
\mathrm{PL}(d)=\mathrm{PL}\left(d_{0}\right)+10 n \log _{10}\left(\frac{d}{d_{0}}\right)+X_{\sigma}
$$

where $n$ is the path loss exponent for a particular frequency band and environment. It is dimensionless and has been assumed to be equal to 3.5 for the NLOS case [21]; $X_{\sigma}$ is a zero mean Gaussian random variable with standard deviation $\sigma$ (in $\mathrm{dB}$ ) taking into account the fluctuations of the signal resulting from the shadowing and $\operatorname{PL}\left(d_{0}\right)$ (in $\mathrm{dB}$ ). The free space path loss is considered at reference distance $d_{0}$ (in $\mathrm{m}$ ) and defined as follows:

$$
\mathrm{PL}\left(d_{0}\right)=10 \log _{10}\left(\frac{4 \pi d_{0}}{\lambda}\right)^{2},
$$

where $\lambda$ is the wavelength (in $\mathrm{m}$ ).
TABLE 3: Power model parameters.

\begin{tabular}{lcc}
\hline Parameters & Description & Values \\
\hline$P_{\text {trans }}$ & $\begin{array}{c}\text { Power RF transceiver per antenna } \\
\text { branch }\end{array}$ & $1.5 \mathrm{~W}$ \\
$\eta$ & Power amplifier efficiency & $50 \%$ \\
$P_{\text {bhl }}$ & Power backhaul & $10 \mathrm{~W}$ \\
$P_{\text {cool }}$ & Power cooling system & $200 \mathrm{~W}$ \\
$P_{\text {rect }}$ & Power rectifier & $50 \mathrm{~W}$ \\
$P_{\mathrm{dsp}}$ & Power signal processing per & $1 \mathrm{~W}$ \\
\hline
\end{tabular}

At the millimeter-wave frequency bands, $\sigma$ is assumed to be equal to $10 \mathrm{~dB}, d_{0}$ equals $1 \mathrm{~m}$, and $\operatorname{PL}\left(d_{0}\right)=68 \mathrm{~dB}$ [24].

The values of the parameters summarized in Table 2 have been derived according to realistic values found in the literature $[2,5,13-20]$. Based on these $5 \mathrm{G}$ parameters, a link budget for the $5 \mathrm{G}$ macrocell is designed in order to characterize the expected performance of the considered system. It considers all the gains and the losses at the transmitter and the receiver and in the propagation medium through the receiver.

3.3. Power Consumption Models. Based on the surveys on energy consumption of cellular networks conducted in [25], the base stations appeared to be the most energy-consuming component of the cellular network, with $80 \%$ of energy required in the network, compared to the mobile stations and the core network. The main objective of the power consumption model used in this study is to determine realistic input parameters in order to have a clear idea of the power consumption of the $5 \mathrm{G}$ wireless networks. Since the simulations focus on the energy efficiency, we implement this model in the deployment tool to assess the power consumption of the network. In Table 3, the power model parameters are summarized.

For the $5 \mathrm{G}$ base station, we assume the power consumption model defined in [26] whereby the macrocell BS consists of six main power consuming components:

(i) Digital signal processing (DSP): used for the digitization of the analog signals and their processing.

(ii) Power amplifier: responsible for the conversion of the DC input power into a significant RF signal.

(iii) Air conditioning: maintains an acceptable temperature for the base station equipment to work smoothly.

(iv) Backhaul link: responsible for communicating the backhaul network with the base station. It can be either a microwave link or a fiber one.

(v) RF transceiver: used for the transmission and reception of the signal at the base station.

(vi) Rectifier: responsible for the $\mathrm{AC}$ to $\mathrm{DC}$ conversion needed by the BS equipment.

The power consumption parameters related to these $5 \mathrm{G}$ base station components are indicated in Table 3. The air conditioning and backhaul power have constant values, while the 


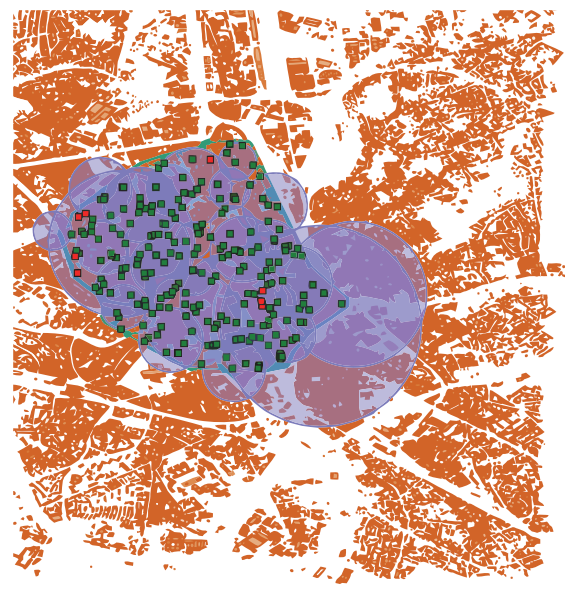

(a) 4G scenario coverage: covered users in green and uncovered users in red

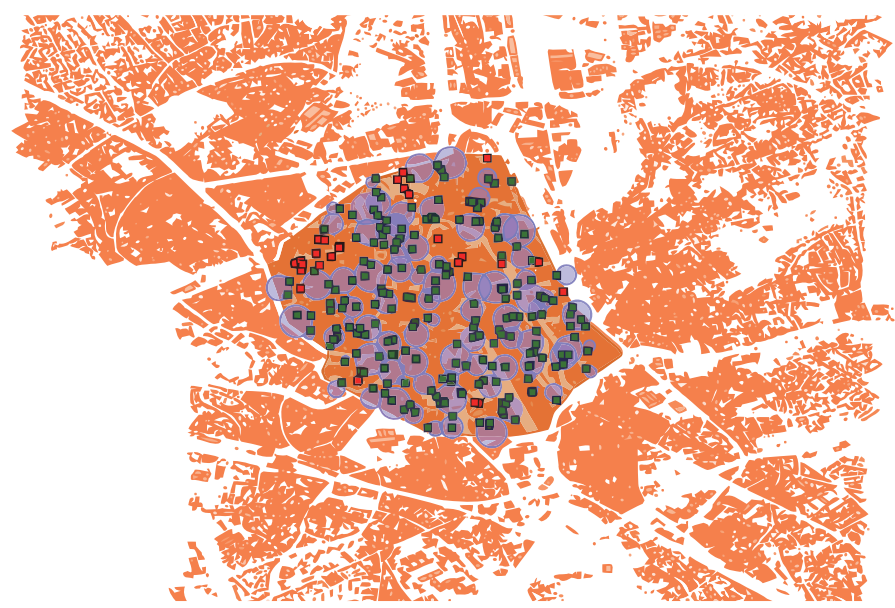

(b) $5 \mathrm{G}$ scenario coverage at $500 \mathrm{MHz}$ : covered users in green and uncovered users in red

FiguRE 4: Example of the 4G and 5G network for the reference scenario.

power consumption of the equipment of the base station (the DSP, the power amplifier, and the RF transceiver) scale with the number of antenna elements. To estimate the total power consumed by these last components, we need to multiply their respective power by the number of antennas. The values in Table 3 are assumed for the 5G BS equipment [27]. For the power consumption of the amplifier, the efficiency $\eta(\%)$ of the power amplifier is used instead. It is defined as the ratio of the RF output power to the electrical input power:

$$
\eta=\frac{P_{t x}}{P_{\mathrm{amp}}}
$$

with $P_{t x}$ being the RF output power of the amplifier unit (in $\mathrm{W}$ ) and $P_{\mathrm{amp}}$ being the electrical input power of the amplifier unit (in W). The total power consumption of the base station is given by the equations, depending on the type of beamforming architecture considered:

$$
\begin{aligned}
P_{\mathrm{DBF}}= & N_{\mathrm{ant}} \cdot\left(P_{\mathrm{trans}}+P_{\mathrm{dsp}}+\eta \cdot P_{\mathrm{amp}}\right)+P_{\text {rect }}+P_{\mathrm{cool}} \\
& +P_{\mathrm{bhl}}, \\
P_{\mathrm{ABF}}= & N_{\mathrm{ant}} \cdot\left(\eta \cdot P_{\mathrm{amp}}\right)+P_{\text {trans }}+P_{\text {rect }}+P_{\mathrm{cool}}+P_{\mathrm{bhl}}, \\
P_{\mathrm{HBF}}= & N_{\mathrm{ant}} \cdot\left(\eta \cdot P_{\mathrm{amp}}\right)+M_{\text {trans }} \cdot P_{\text {trans }}+P_{\mathrm{dsp}}+P_{\text {rect }} \\
& +P_{\mathrm{cool}}+P_{\mathrm{bhl}},
\end{aligned}
$$

with $\mathrm{DBF}, \mathrm{ABF}$, and $\mathrm{HBF}$ standing for digital beamforming, analog beamforming and hybrid beamforming, respectively; $N_{\text {ant }}$ is the number of BS antenna elements, $M_{\text {trans }}$ is the number of RF transceivers used, $P_{\text {trans }}$ is the power consumption of the RF transceiver unit (in W), $P_{\mathrm{dsp}}$ is the power consumption of the DSP unit (in W), $\eta$ is the amplifier unit efficiency, $P_{\mathrm{amp}}$ is the electrical input power of the amplifier unit (in W), $P_{\text {rect }}$ is the power consumption of the rectifier unit (in W), $P_{\text {cool }}$ is the power consumption of the air conditioning (in W), and $P_{\text {bhl }}$ is the power consumption of the backhaul link (in W).
3.4. Energy Efficiency Metrics. Deciding which base station provides the better energy efficiency is not simple since various parameters are taken into account (bandwidth, capacity, covered users, etc.). For a better comparison, we will make use of an energy efficiency (EE) metric that takes into account multiple network performance parameters such as the bandwidth, the bit rate, the coverage, and the capacity. The energy efficiency (EE) metric in this study is defined by the following equation [4]:

$$
\mathrm{EE}=\frac{A \cdot B \cdot U}{P_{\mathrm{el}}}
$$

where $A$ is the area covered by the BS (in $\mathrm{km}^{2}$ ), $U$ is the number of served users, $B$ is the bit rate based on the base station (in Mbps), and $P_{\mathrm{el}}$ is the power consumption of the base station. The higher the EE value, the more energy efficient the network.

\section{Results}

4.1. Network Performance Comparison without Beamforming. In this section, we evaluate the network performance obtained with the $4 \mathrm{G}$ reference scenario and the $5 \mathrm{G}$ scenario II.a in Table 1, whereby beamforming is not used at all (neither on BS nor on MS side). For a good comparison of these scenarios, the location of the base stations in the considered area is chosen such that the compared networks serve more than $96 \%$ of the users. Figure 4 shows an example of a $4 \mathrm{G}$ and $5 \mathrm{G}$ network.

Figure 5 shows that the $5 \mathrm{G}$ scenario requires more BSs than the $4 \mathrm{G}$ reference network (92 BS versus $33 \mathrm{BS}$ ). This is explained by the fact that the range of the cell in $5 \mathrm{G}$ is $39.6 \%$ smaller than the $4 \mathrm{G}$ ones based on the assumptions of this study (Figure 4).

However, 5G base stations are less power consuming than $4 \mathrm{G}$ ones. There is a reduction of almost $50 \%$ in power consumption, despite the higher number of base stations in the $5 \mathrm{G}$ networks. In fact, the $5 \mathrm{G}$ scenario II.a consumes 

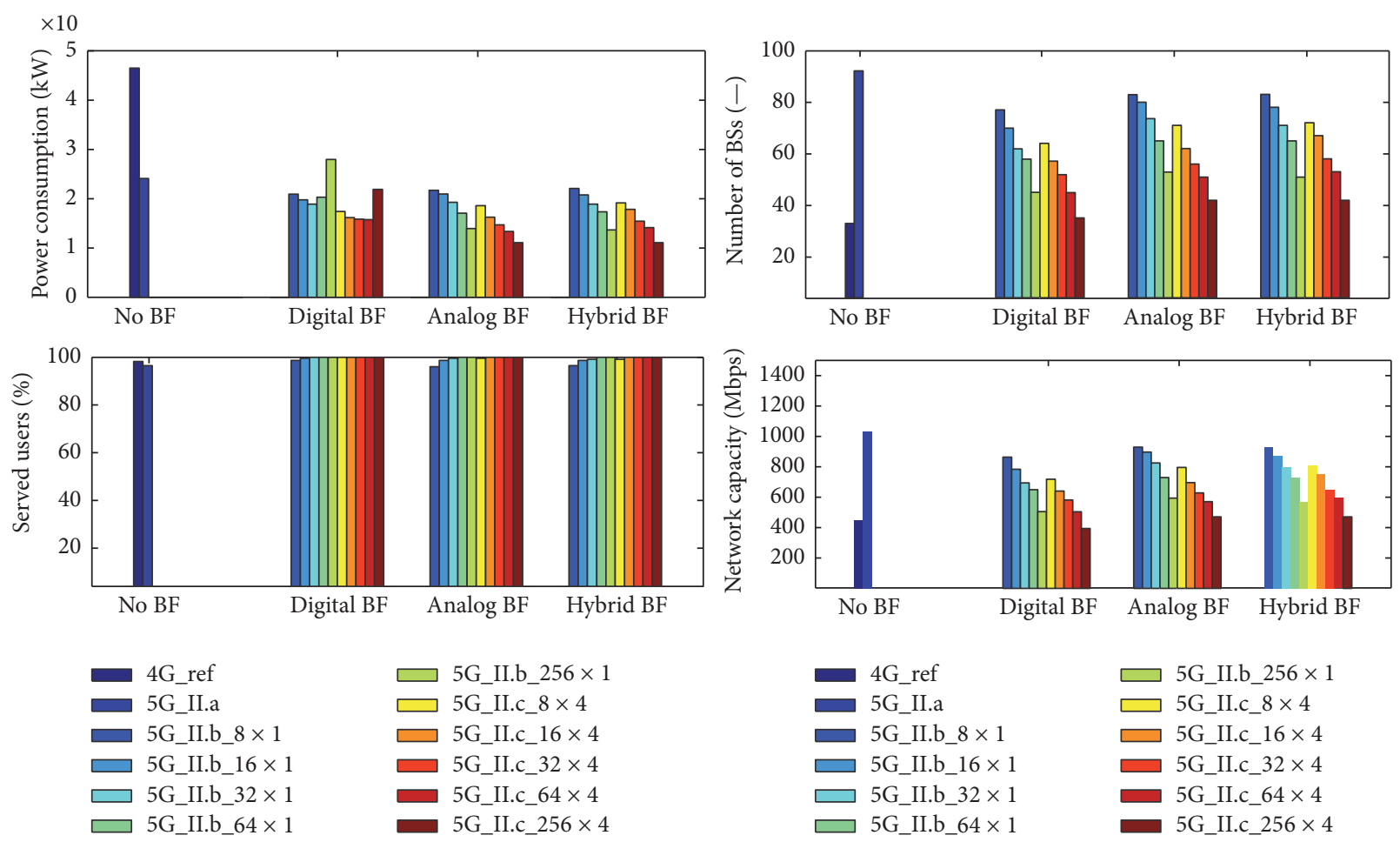

FIGURE 5: Comparison of different parameters when beamforming is used: number of base stations, percentage of served users, power consumption, and capacity offered by the network.

$24.1 \mathrm{~kW}$ (Figure 5) compared to the $46.5 \mathrm{~kW}$ consumed by the $4 \mathrm{G}$ reference network. This can be attributed to the new technologies developed by the manufacturers to build low-cost and power efficient RF front-end components [28]. Table 3 shows that, in 5G, the rectifier, the RF transceiver unit, the backhaul link, and the DSP consume 50\%, 87\%, 87.5\%, and $92 \%$ less power, respectively, compared to the power consumption of the $4 \mathrm{G} \mathrm{RF}$ components assumed in [4].

For the entire network capacity (based on the BS), the considered 5G scenario offers higher capacity than the $4 \mathrm{G}$ network, $1032.6 \mathrm{Mbps}$ for $5 \mathrm{G}$ scenario II.a, while the $4 \mathrm{G}$ offers $449.5 \mathrm{Mbps}$, as shown in Figure 5. This is because the 5G networks use more base stations compared to the $4 \mathrm{G}$ ones, as explained above (Figure 5).

Based on the energy efficiency metric defined in Section 3.3, whereby all the above parameters are combined, the $4 \mathrm{G}$ reference network is less energy efficient since it does have a smaller EE value compared to the considered $5 \mathrm{G}$ scenario $\left(14.6\left[\mathrm{~km}^{2} \cdot \mathrm{Mbps} / \mathrm{W}\right]\right.$ for $4 \mathrm{G}$ and $30.6\left[\mathrm{~km}^{2} \cdot \mathrm{Mbps} / \mathrm{W}\right]$ for $5 \mathrm{G}$ scenarios II.a). This better performance in terms of EE is sustained by the power consumption of the $5 \mathrm{G}$ network, that is, $50 \%$ lower than the $4 \mathrm{G}$ reference network (Figure 6).

4.2. Influence of the Use of Beamforming. Here, we examine the behavior of the 5G scenarios II.b and II.c described in Section 3.3, whereby beamforming is utilized (Table 4). The results of the simulations are presented in Figure 5.

Based on the digital beamforming architecture, where a transceiver is behind each antenna element, the results show that the more the antenna elements are used, the better the

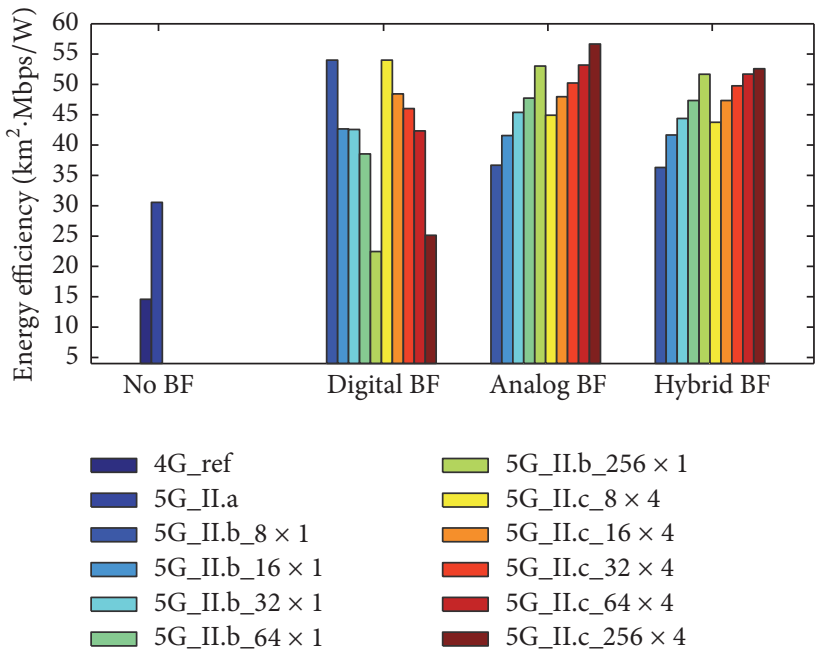

FIGURE 6: Energy efficiency parameter for different beamforming architectures.

coverage provided by the network is. Figure 5 shows that the $5 \mathrm{G}$ networks require more base stations than the ones obtained with the $4 \mathrm{G}$ reference scenario: $+75.4 \%$ for scenario II.b $64 \times 1,+36.4 \%$ for scenario II.b $256 \times 1,+36.1 \%$ for scenario II.c $64 \times 4$, and $+6.2 \%$ for scenario II.c $256 \times 4$. The multiple antennas provide additional gains and make it possible to overcome the millimeter waves propagation constraints. This results into a higher MAPL that gives rise to a higher value of the cell range (e.g., when using $256 \times 4$, the range increases by 
TABLE 4: Simulation results (95 percentile).

\begin{tabular}{|c|c|c|c|c|c|c|c|}
\hline Beamforming & Scenario & $\mathrm{PC}[\mathrm{kW}]$ & \# BS [-] & $\begin{array}{c}\text { Served } \\
\text { users [\%] }\end{array}$ & $\begin{array}{c}\text { Area } \\
\text { coverage [\%] }\end{array}$ & $\begin{array}{c}\text { Network capacity } \\
{[\mathrm{Mbps}]}\end{array}$ & $\begin{array}{c}\text { Energy efficiency } \\
{\left[\mathrm{km}^{2} \cdot \mathrm{Mbps} / \mathrm{W}\right]}\end{array}$ \\
\hline \multirow{2}{*}{ No beamforming } & 4G_ref & 46.5 & 33 & 98.8 & 98.2 & 449.5 & 14.6 \\
\hline & 5G_II.a & 24.1 & 92 & 96.5 & 45.9 & 1032.6 & 30.6 \\
\hline \multirow{10}{*}{ Digital beamforming } & 5G_II.b_8x1 & 20.9 & 77 & 98.7 & 65.6 & 863.5 & 53.9 \\
\hline & 5G_II.b_16x1 & 19.7 & 70 & 99.6 & 70.4 & 784 & 42.6 \\
\hline & 5G_II.b_32x1 & 18.9 & 62 & 100 & 75.5 & 694.4 & 42.6 \\
\hline & 5G_II.b_64x1 & 20.3 & 58 & 100 & 78.5 & 649.6 & 38.5 \\
\hline & 5G_II.b_256xl & 27.9 & 45 & 100 & 80.9 & 505.1 & 22.5 \\
\hline & 5G_II.c_8x4 & 17.4 & 64 & 100 & 85.2 & 717.9 & 53.9 \\
\hline & 5G_II.c_16x4 & 16.2 & 57 & 100 & 79.6 & 640.6 & 48.4 \\
\hline & 5G_II.c_32x4 & 15.8 & 52 & 100 & 81.6 & 582.4 & 46 \\
\hline & 5G_II.c_64x4 & 15.7 & 45 & 100 & 86.2 & 504 & 42.3 \\
\hline & 5G_II.c_256x4 & 21.9 & 35 & 100 & 91.4 & 394.2 & 25.1 \\
\hline \multirow{10}{*}{ Analog beamforming } & 5G_II.b_8x1 & 21.7 & 83 & 96 & 58.1 & 929.6 & 36.7 \\
\hline & 5G_II.b_16x1 & 20.9 & 80 & 98.7 & 64.1 & 897.1 & 41.6 \\
\hline & 5G_II.b_32x1 & 19.3 & 74 & 99.6 & 69.4 & 825.4 & 45.4 \\
\hline & 5G_II.b_64x1 & 17.1 & 65 & 100 & 72.8 & 729.1 & 47.7 \\
\hline & 5G_II.b_256x1 & 13.9 & 53 & 100 & 81.2 & 593.6 & 53 \\
\hline & 5G_II.c_8x4 & 18.6 & 71 & 99.6 & 68.6 & 796.3 & 44.9 \\
\hline & 5G_II.c_16x4 & 16.2 & 62 & 100 & 73.0 & 695.5 & 47.9 \\
\hline & 5G_II.c_32x4 & 14.7 & 56 & 100 & 76.5 & 628.3 & 50.2 \\
\hline & 5G_II.c_64x4 & 13.4 & 51 & 100 & 81.1 & 571.2 & 53.2 \\
\hline & 5G_II.c_256x4 & 11.1 & 42 & 100 & 81.9 & 470.4 & 56.6 \\
\hline \multirow{10}{*}{ Hybrid beamforming } & 5G_II.b_8x1 & 22.1 & 83 & 96.5 & 58.2 & 930.7 & 36.3 \\
\hline & 5G_II.b_16x1 & 20.7 & 78 & 98.7 & 65.3 & 874.7 & 41.6 \\
\hline & 5G_II.b_32x1 & 18.9 & 71 & 99.1 & 69.2 & 796.3 & 44.4 \\
\hline & 5G_II.b_64x1 & 17.3 & 65 & 100 & 73.3 & 729.1 & 47.3 \\
\hline & 5G_II.b_256x1 & 13.6 & 51 & 100 & 80.4 & 571.2 & 51.7 \\
\hline & 5G_II.c_8x4 & 19.1 & 72 & 99.1 & 68.2 & 807.5 & 43.8 \\
\hline & 5G_II.c__16x4 & 17.8 & 67 & 100 & 73.2 & 751.5 & 47.3 \\
\hline & 5G_II.c_32x4 & 15.4 & 58 & 100 & 76.9 & 650.7 & 49.8 \\
\hline & 5G_II.c_64x4 & 14.1 & 53 & 100 & 80 & 594.7 & 51.7 \\
\hline & 5G_II.c_256x4 & 11.1 & 38 & 100 & 80.6 & 470.4 & 52.6 \\
\hline
\end{tabular}

$15.17 \%)$. So, when beamforming is applied at both sides, the number of base stations of $5 \mathrm{G}$ networks is approaching the $4 \mathrm{G}$ ones, specifically when the number of antenna elements is increasing.

Beamforming improves the coverage of the $5 \mathrm{G}$ network, in terms of both the area and the served users thanks to the additional gains provided by the multiple antenna elements used. The performance approaches the $4 G$ ones (99\%) in terms of served users: $99.6 \%$ of the users are covered in scenario II.b $(16 \times 1)$ and $100 \%$ in scenario II.c $(256 \times 4)$. In terms of coverage, beamforming improves the coverage of the $5 \mathrm{G}$ network by $99 \%$ (DBF scenario II.b $256 \times 4$ ), in comparison with the $5 \mathrm{G}$ scenario II.a, whereby beamforming is not used.
However, 4G networks still provide better performance: $98 \%$ of the considered area is covered while $5 \mathrm{G}$ covers $91.4 \%$ of the considered area.

In terms of power consumption (Figure 5), when multiple antennas are used on the BS side, the $5 \mathrm{G}$ networks consume almost $25 \%$ less power (HBF scenario II.b 256x4) than the $4 \mathrm{G}$ reference network. This is realized by the technology scaling that allows the manufacturing of very low-power RF frontends components used in the RF circuits (transceiver, ADC, DAC, mixers, etc.).

When considering a RF beamforming architecture, we obtain similar results (compared to digital beamforming) in terms of number of base stations, served users, and coverage 
area. Digital beamforming performances are better than the RF beamforming one: $91.4 \%$ of the considered area is covered and $100 \%$ of the users are served (scenario II.c 256x4). These performances are achieved since the beamforming function is implemented in the baseband stage where high-speed digital signal processors (DSP) compute complex algorithms that determine the required phase and amplitude of the transmitted signal. This makes the DBF more flexible as it is easy to reprogram the algorithms. However, there is a price to pay, in terms of the power consumption and the cost of implementation, that limits the scalability of the architecture. In fact, the digital beamforming consumes 2 times more power to achieve its performance (Figure 5), compared to $\mathrm{RF}$ beamforming. The increase in power consumption is mainly due to the excessive number of RF transceivers and the Analog to Digital Converters (ADC) and Digital to Analog Converters (DAC) required, while the analog beamforming uses only one RF chain to drive the antenna arrays. In addition, the analog beamforming which presents attractive power consumption results has some drawbacks: the phase shifters used in the RF domain have nonideal characteristics that lead to the noise and losses, preventing this architecture from providing similar performances as digital one.

It then becomes obvious to consider a trade-off between the achievement of better performances while meeting the power consumption requirements. For this purpose, a hybrid architecture is proposed $[12,29]$. With this architecture, the MIMO precoding and beamforming are performed on the baseband and RF sides, respectively, to allow reasonable number of RF chains required by using 2 to 8 transceivers [30]. In this study, we consider a hybrid architecture with two transceivers. Figure 5 shows that the results are similar (compared to digital beamforming) in terms of number base stations, coverage area, and served users. The requirement of power consumption is also met in hybrid beamforming architecture. It is 2 times less power consuming than the digital beamforming (scenarios II.b 256x1 and II.c 256x4).

(Figure 6) shows that the scenarios II.b and II.c are presenting higher energy efficiency, irrespective of the beamforming architecture used, compared to the $4 \mathrm{G}$ reference network: $14.6\left[\mathrm{~km}^{2} \cdot \mathrm{Mbps} / \mathrm{W}\right]$ for $4 \mathrm{G}, 22.5\left[\mathrm{~km}^{2} \cdot \mathrm{Mbps} / \mathrm{W}\right]$ for $5 \mathrm{G}$ scenario II.b $256 \mathrm{x} 1$, and $25.1\left[\mathrm{~km}^{2} \cdot \mathrm{Mbps} / \mathrm{W}\right]$ for $5 \mathrm{G}$ scenario II.b 256x4. However, the RF and hybrid beamforming architectures are more energy efficient than the digital beamforming: $56.6\left[\mathrm{~km}^{2} \cdot \mathrm{Mbps} / \mathrm{W}\right]$ for ABF $256 \mathrm{x} 4$, $52.6\left[\mathrm{~km}^{2} \cdot \mathrm{Mbps} / \mathrm{W}\right]$ for HBF $256 \mathrm{x} 4$, and $25.1\left[\mathrm{~km}^{2} \cdot \mathrm{Mbps} / \mathrm{W}\right]$ for DBF $256 \times 4$. For the same user coverage (100\%), the DBF is performing better in terms of number of base stations; it requires $17 \%$ less $\mathrm{BS}$ than $\mathrm{ABF}$ and $\mathrm{HBF}$, respectively, for scenarios II.c $256 \times 4$. Though the RF beamforming architecture is the most energy efficient architecture, based on the considered EE parameter, it does not appear to be the best candidate since it does not cover the considered area as good as the DBF ( $82 \%$ of area covered for ABF $256 \times 4$ and $91 \%$ of area covered for DBF 256x4). This worse performance in terms of area of coverage may lead to outages during the mobility of the users within the considered area. So, a tradeoff needs to be considered between the two architectures. The hybrid beamforming architecture would be recommended instead since it achieves acceptable performances at lowpower consumption, without embarking too many RF frontends components.

\section{Conclusions}

In this study, we evaluate the performance of the $5 \mathrm{G}$ cellular network using a capacity-based deployment tool. The tool has been proposed to simulate a realistic network that responds to the instantaneous bit rate required by the users, in the considered area of Ghent, Belgium. Various 5G scenarios have been considered for the simulations, whereby suitable link budget parameters, millimeter-wave path loss models, power consumption models, and energy efficiency metric have been used. Based on the results of the simulations, we show that the $5 \mathrm{G}$ scenario whereby beamforming is not implemented requires much more BSs than the $4 \mathrm{G}$ reference scenario. It is $50 \%$ less power consuming and provides 2 times more capacity than $4 \mathrm{G}$. However, it is not a good candidate for network planning because of the poor coverage (46\%) of the considered area.

We further extended the analysis to consider multiple antennas and the use of beamforming at both the BS and the MS. The results show that $5 \mathrm{G}$ networks supporting beamforming are 3 times more energy efficient than $4 G$ networks, based on the defined energy efficiency parameter. The same $4 \mathrm{G}$ network coverage performances are achieved with 4 times less power consumption (scenarios $\mathrm{ABF}$ and HBF 256x4). However, among the beamforming architectures considered, the digital beamforming presents better performance than the other two architectures but it does not meet the power consumption requirements. We showed that a trade-off was needed to provide better performances at lower power consumption. This can be achieved with the hybrid beamforming architecture which provides similar results in terms of coverage area, served users, and number of base stations as digital beamforming, while consuming two times less power. So, the hybrid beamforming architecture is a better alternative to digital beamforming to design and deploy $5 \mathrm{G}$ networks.

\section{Competing Interests}

The authors declare that there is no conflict of interests regarding the publication of this paper.

\section{References}

[1] L. B. Le, V. Lau, E. Jorswieck et al., "Enabling 5G mobile wireless technologies," EURASIP Journal on Wireless Communications and Networking, vol. 2015, article 218, 2015.

[2] A. Gupta and R. K. Jha, "A survey of 5G network: architecture and emerging technologies," IEEE Access, vol. 3, pp. 1206-1232, 2015.

[3] R. Q. Hu and Y. Qian, "An energy efficient and spectrum efficient wireless heterogeneous network framework for $5 \mathrm{G}$ systems," IEEE Communications Magazine, vol. 52, no. 5, pp. 94101, 2014

[4] M. Deruyck, W. Joseph, E. Tanghe, and L. Martens, "Reducing the power consumption in LTE-Advanced wireless access 
networks by a capacity based deployment tool," Radio Science, vol. 49, no. 9, pp. 777-787, 2014.

[5] T. Bogale and L. Le, "Massive MIMO and mmWave for 5G Wireless HetNet: potential benefits and challenges," IEEE Vehicular Technology Magazine, vol. 11, no. 1, pp. 64-75, 2016.

[6] A. Osseiran, F. Boccardi, V. Braun et al., "Scenarios for 5G mobile and wireless communications: the vision of the METIS project," IEEE Communications Magazine, vol. 52, no. 5, pp. 2635, 2014.

[7] W. Roh, J.-Y. Seol, J. Park et al., "Millimeter-wave beamforming as an enabling technology for $5 \mathrm{G}$ cellular communications: theoretical feasibility and prototype results," IEEE Communications Magazine, vol. 52, no. 2, pp. 106-113, 2014.

[8] Huawei, “5g: a technology vision," 2015.

[9] Nokia, “Ten key rules of 5g deployment," 2015.

[10] T. S. Rappaport, S. Sun, R. Mayzus et al., "Millimeter wave mobile communications for 5G cellular: it will work!," IEEE Access, vol. 1, pp. 335-349, 2013.

[11] S. Path, A Straight Path Towards 5G, Straight Path Communications Inc, 2015.

[12] F. W. Vook, A. Ghosh, and T. A. Thomas, "MIMO and beamforming solutions for 5G technology," in Proceedings of the IEEE MTT-S International Microwave Symposium (IMS '14), pp. 1-4, Tampa, Fla, USA, June 2014.

[13] E. Grass, “Towards 100 gbps: ultra-high spectral efficiency using massive mimo with 3d antenna configurations," 2013.

[14] J. Chen, Advanced architectures of efficient mm-wave cmos wireless transmitters [Ph.D. thesis], 2013.

[15] A. Puglielli, A. Townley, G. LaCaille et al., "Design of energyand cost-efficient massive MIMO arrays," Proceedings of the IEEE, vol. 104, no. 3, pp. 586-606, 2015.

[16] F. Khan and Z. Pi, "mmWave mobile broadband (MMB): unleashing the 3-300GHz spectrum," in Proceedings of the IEEE Sarnoff Symposium (SARNOFF '11), May 2011.

[17] Z. Pi, J. Choi, and R. Heath, "Millimeter-wave gigabit broadband evolution toward 5G: fixed access and backhaul," IEEE Communications Magazine, vol. 52, no. 9, pp. 88-95, 2015.

[18] A. Rahimian and F. Mehran, "RF link budget analysis in urban propagation microcell environment for mobile radio communication systems link planning," in Proceedings of the International Conference on Wireless Communications and Signal Processing (WCSP '11), pp. 1-5, IEEE, Nanjing, China, November 2011.

[19] S. Rajagopal, S. Abu-Surra, Z. Pi, and F. Khan, "Antenna array design for multi-Gbps mmWave mobile broadband communication," in Proceedings of the Annual IEEE Global Telecommunications Conference: "Energizing Global Communications" (GLOBECOM '11), Houston, Tex, USA, December 2011.

[20] C. Dehos, J. González, A. Domenico, D. Kténas, and L. Dussopt, "Millimeter-wave access and backhauling: the solution to the exponential data traffic increase in $5 \mathrm{G}$ mobile communications systems?" IEEE Communications Magazine, vol. 52, no. 9, pp. 88-95, 2014.

[21] G. R. Maccartney, T. S. Rappaport, M. K. Samimi, and S. Sun, "Millimeter-wave omnidirectional path loss data for small cell 5G channel modeling," IEEE Access, vol. 3, pp. 1573-1580, 2015.

[22] S. Sun, T. A. Thomas, T. S. Rappaport, H. Nguyen, I. Z. Kovacs, and I. Rodriguez, "Path loss, shadow fading, and line-of-sight probability models for $5 \mathrm{~g}$ urban macro-cellular scenarios," in Proceedings of the IEEE Globecom Workshops (GC Wkshps '15), pp. 1-7, San Diego, CA, USA, December 2015.
[23] A. Sulyman, A. Nassar, M. Samimi, G. Maccartney, T. Rappaport, and A. Alsanie, "Radio propagation path loss models for $5 \mathrm{G}$ cellular networks in the $28 \mathrm{GHZ}$ and $38 \mathrm{GHZ}$ millimeterwave bands," IEEE Communications Magazine, vol. 52, no. 9, pp. 78-86, 2014.

[24] T. S. Rappaport, G. R. MacCartney, M. K. Samimi, and S. Sun, "Wideband millimeter-wave propagation measurements and channel models for future wireless communication system design," IEEE Transactions on Communications, vol. 63, no. 9, pp. 3029-3056, 2015.

[25] A. Fehske, G. Fettweis, J. Malmodin, and G. Biczok, "The global footprint of mobile communications: the ecological and economic perspective," IEEE Communications Magazine, vol. 49, no. 8, pp. 55-62, 2011.

[26] M. Deruyck, E. Tanghe, W. Joseph et al., "Model for power consumption of wireless access networks," IET Science, Measurement and Technology, vol. 5, no. 4, pp. 155-161, 2011.

[27] MAMMOET, "Massive mimo for efficient transmission: deliverables 1.1, systems scenarios and requirements specifications," 2014.

[28] E. Larsson, O. Edfors, F. Tufvesson, and T. Marzetta, "Massive MIMO for next generation wireless networks," IEEE Transactions on Electromagnetic Compatibility, vol. 52, no. 4, pp. 186195, 2014.

[29] D. Muirhead, M. A. Imran, and K. Arshad, "Insights and approaches for low-complexity 5G small-cell base-station design for indoor dense networks," IEEE Access, vol. 3, pp. 15621572, 2015.

[30] R. W. Heath, N. Gonzalez-Prelcic, S. Rangan, W. Roh, and A. M. Sayeed, "An overview of signal processing techniques for millimeter wave MIMO systems," IEEE Journal of Selected Topics in Signal Processing, vol. 10, no. 3, pp. 436-453, 2016. 

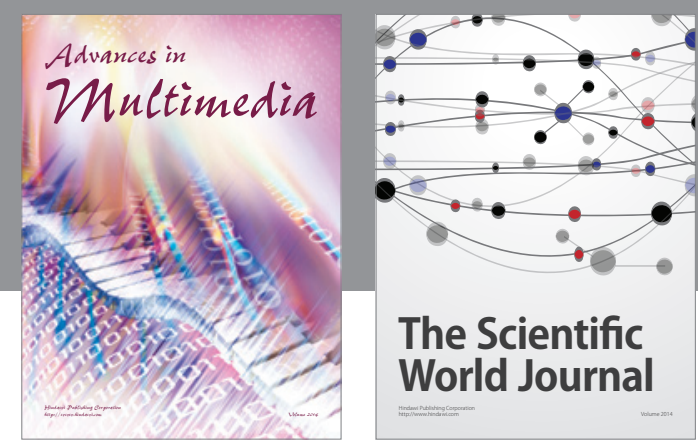

The Scientific World Journal
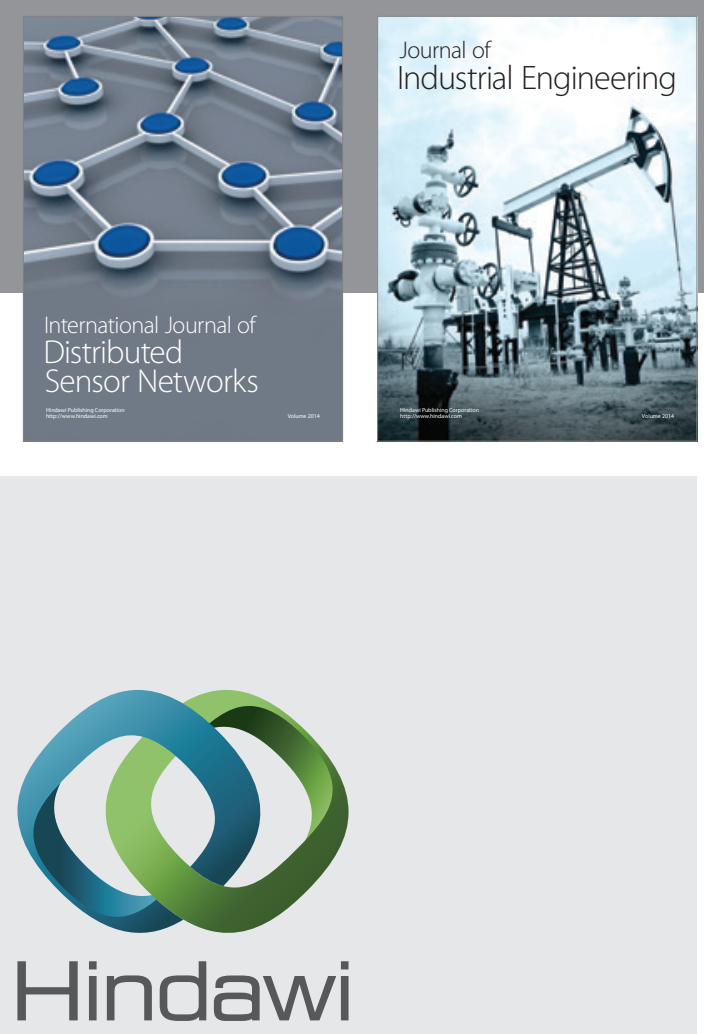

Submit your manuscripts at

https://www.hindawi.com

\section{Computer Networks} and Communications
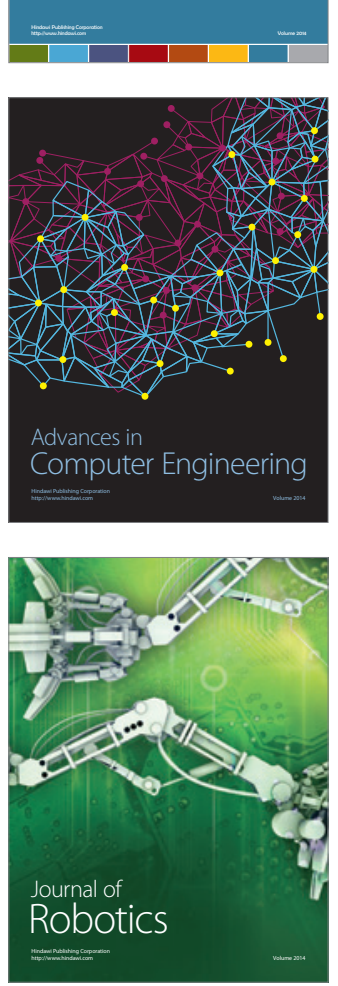
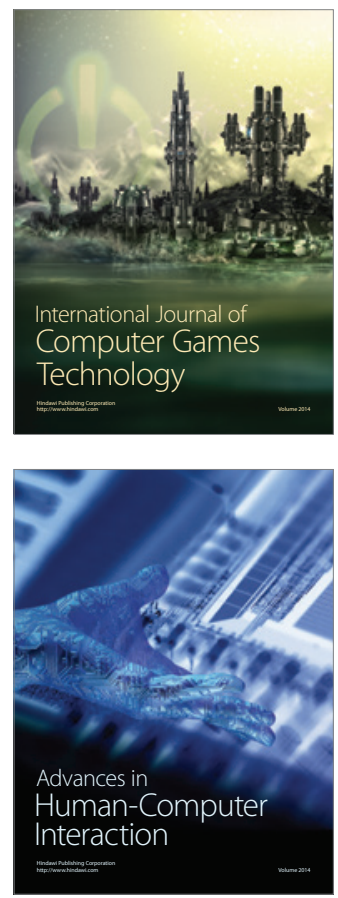
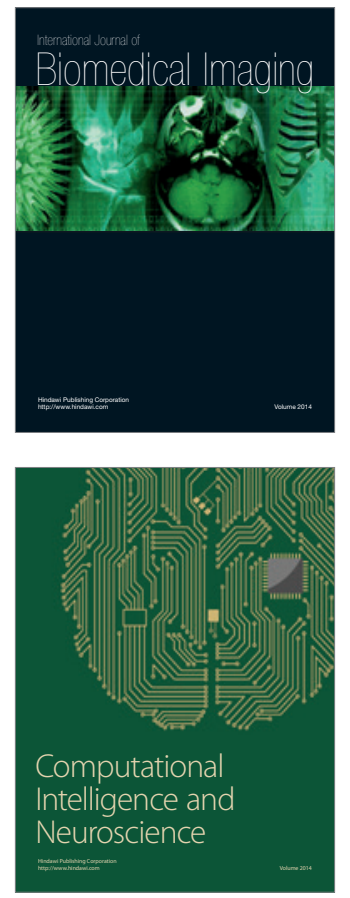
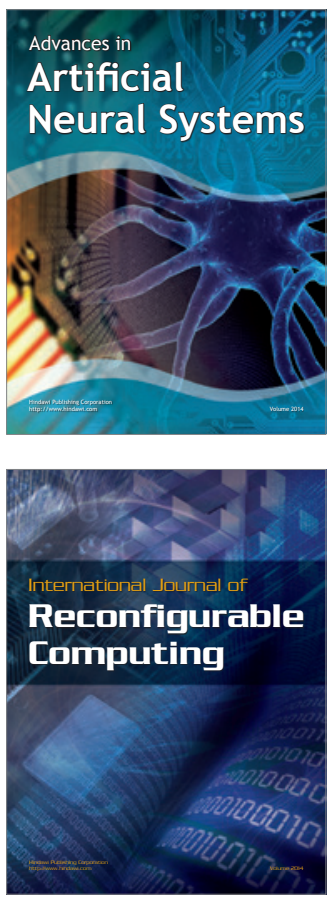
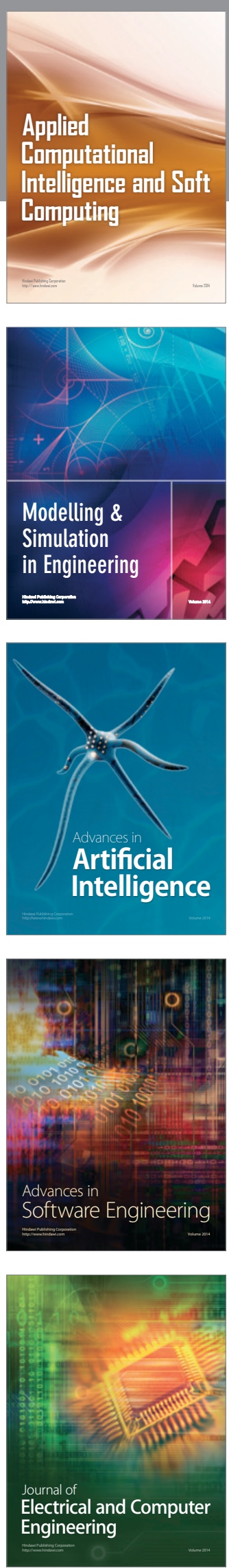